\title{
Adaptive Coding in Visual Cortical Circuits
}

\author{
Bryan J. Hansen and Valentin Dragoi* \\ University of Texas-Houston Medical School \\ Houston, TX 77030, USA \\ *valentin.dragoi@uth.tmc.edu
}

\begin{abstract}
Understanding the rules by which brain networks represent incoming stimuli in population activity to influence the accuracy of behavioral responses remains one of the deepest mysteries in neuroscience. We have embarked on a set of projects to investigate the real-time operation of multiple neuronal networks and their capacity to undergo adaptive changes and plasticity. What are the fundamental units of network computation and the principles that govern their relationship with behavior? By employing state-of-theart electrophysiological techniques we were able to record from large pools of cells in the non-human primate brain while animals performed a fixation task. We found that spatiotemporal correlations between neurons could act as an active 'switch' to control network performance in real time by modulating the communication between neurons. We believe that 'cracking' the mysteries of the population code will offer unique insight into a network-based mechanistic explanation of behavior and new therapeutic solutions to cure brain dysfunction.
\end{abstract}

\section{Introduction}

A fundamental feature of cortical neurons is their ability to rapidly adapt to changes in incoming stimuli. Several lines of evidence indicate that cortical neurons dynamically change their responses and selectivity to match the changes in the statistics of the input stimuli. Whereas our understanding of information processing at the single neuron level has substantially improved over the past three decades, how populations of brain cells encode information remains largely unknown. In particular, understanding how adaptation changes information processing by individual neurons and networks is essential is essential for understanding the relationship between efficient sensory coding and behavior. We have recently performed neurophysiological investigations to examine how rapid adaptation at the time scale of visual fixation impacts information processing in networks of brain cells.

\section{Background}

\subsection{Rapid adaptation}

Adaptation is a ubiquitous phenomenon in sensory processing. The time-scale of adaptation can range from hundreds of milliseconds to several days. In normal visual processing, adaptation occurs along many stimulus dimensions such as luminance, contrast, orientation, spatial frequency, direction of motion, color, and curvature and shape. In the orientation domain, adaptation has been shown to influence the signaling capabilities of neurons in primary visual cortex (V1). For example, adaptation to an oriented grating induces a repulsive shift in the preferred of individual V1 neurons and changes orientation selectivity when the adapting grating is similar to the cell's optimal orientation [1,2].

Examining rapid adaptation in visual cortex is important for understanding how individual neurons and local circuits change their coding properties in real-time. 
An important perceptual effect of adaptation is the change in orientation discrimination performance. Both perceptual studies and physiological recordings in V1 have demonstrated that adaptation improves the capacity of neurons to signal small differences between stimuli such that orientation discrimination is improved $[1,3,4]$. However, it remains unclear whether and how adaptation influences the information encoded in population activity.

\subsection{Cortical layers}

A fundamental issue in our understanding of brain circuits is how networks in different layers of the cerebral cortex encode information. Cortical layers are ubiquitous structures throughout neocortex $[5,6]$ that consist of highly recurrent local networks that communicate among each other to possibly influence the information encoded in population activity. In recent years, significant progress has been made in our understanding of the differences in response properties of neurons across cortical layers $[7,8]$, yet there is still a great deal to learn about whether and how neuronal populations encode information in a layer-specific manner.

\subsection{Neural synchronization}

It has been proposed that one way in which networks of cells can efficiently process information about incoming stimuli is through synchronization between the spiking activity of individual neurons and local population activity (measured as local field potentials, LFPs). In visual cortex, it has been found that neuronal groups exhibit strong responses in the gamma-band frequency $(30-80 \mathrm{~Hz})[9,10,11]$, and that single neurons synchronize their responses with the local population activity [12].

Synchronization in visual cortex, particularly in the gamma-band, has been found to be critically involved in sensory processing $[9,11,13]$, grouping $[9,11]$, attention $[10,14,15]$, working memory [16], and behavioral reaction times [15].

\section{Experimental methods}

\subsection{Identification of cortical layers.}

To identify cortical layers, we measured the evoked response potentials (ERPs) of LFPs across equally-spaced contacts (Fig. $1 \mathrm{~b}$ left, inter-contact distance $=100 \mu \mathrm{m}$ ) in response to a full-field flashed stimulus.

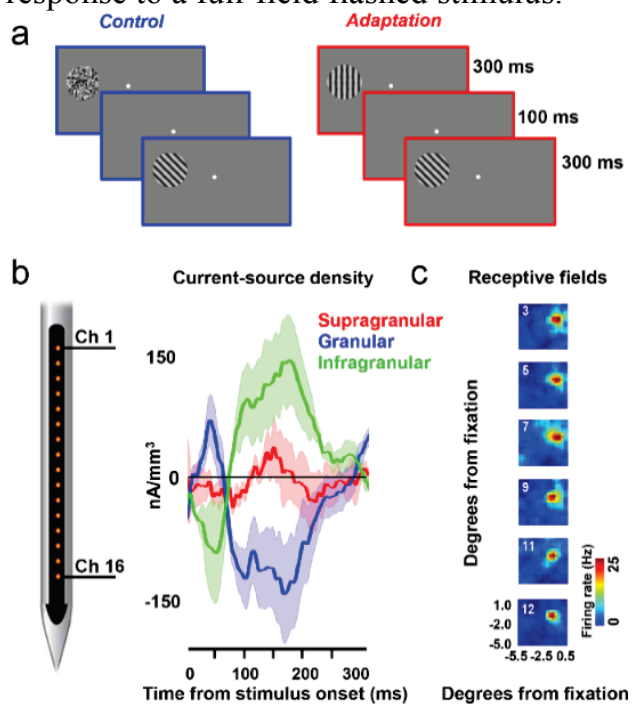

Fig. 1. Rapid adaptation across cortical layers in V1. (a) Schematic description of the orientation adaptation protocol. (b) Multicontact laminar electrodes were used to record neuronal activity across cortical depth (left). Current-source density analysis (based on the 2nd spatial derivate of the LFP time-series) was used to identify the polarity inversion accompanied by the sink-source configuration at the base of the granular layer. The CSD traces represent the average of those contacts assigned to a given layer. (c) Receptive fields across contacts were mapped using oriented stimuli presented in random patches. Firing rates for each neuron are calculated independently at $5 \mathrm{~ms}$ intervals and the maximum firing rates (shown as red) were used to computed the centroid for each time delay.

We then performed current-source density (CSD; Fig. 1b right) analysis of the LFP time-series to identify the polarity inversion accompanied by the sink-source configuration at the base of layer 4 (the sink is inside layer 4 , subsequently referred 
to as the granular layer $[5,6,7,8,17]$. We observed that cells recorded on laminar probes had highly overlapping receptive fields (Fig. 1c) and highly similar preferred orientations (PO) (e.g., the difference in PO for over $58 \%$ of the pairs of neurons was within $0^{\circ}-10^{\circ}$ range, $\mathrm{p}<0.01$, Wilcoxon signed-rank test).
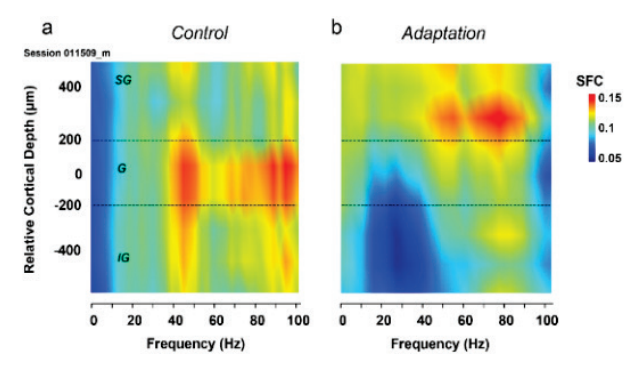

Fig. 2. Example of synchronization, measured as spike-field coherence (SFC) as a function of frequency, across cortical layers during control (left) and adaptation (right). During the presentation of the control stimuli there is an increase in gamma activity in the granular $(\mathrm{G})$ layer. Adaptation increases SFC specifically in the supragranular (SG) layer.

3.2. Measures of synchronization. We used multi-taper spectral analysis to compute spike-field coherence (SFC), which measures the degree of synchronization between individual neurons and local population activity (LFPs) for each specific frequency band. In general, the coherence between two signals ( $x$ and $y$ ) recorded at different sites is a complex quantity whose magnitude is a measure of the phase synchrony for frequency $f$. Coherence is an absolute value that varies between 0 and 1 (e.g., a value of 1 indicates a perfect phase relationship between the firing of the spikes to the fluctuations of the LFP). Coherence is defined as:

$$
C_{x y}(f)=S_{y x}(f) /\left(S_{x x}(f) S_{y y}(f)\right)^{0.5}
$$

where $S_{x x}(f)$ and $S_{y y}(f)$ represent the autospectra and $S_{y x}$ the cross-spectrum of the two signals $x$ and $y$.

\subsection{Neuronal correlations.}

Multiple single-unit recordings were performed from V1 of two fixating monkeys (Macaca mulatta). Stimuli were presented such as to cover the center of the neurons' receptive fields. In control trials, movie strips were presented for $\sim 1.86 \mathrm{~s}$ (16 orientations x 7 repeats at $60 \mathrm{~Hz}$; random spatial phase). In adaptation trials, movies were preceded by a $400-\mathrm{m}$ grating of fixed orientation. The Pearson correlation coefficient of spike counts, $R_{s c}$, of two cells is defined as:

$$
R_{s c}=\frac{\sum_{i=1}^{N}\left(r_{1}^{i}-\overline{r_{1}}\right) \cdot\left(r_{2}^{i}-\overline{r_{2}}\right)}{\sigma_{1} \cdot \sigma_{2}}
$$

where $N$ is the number of trials, $r_{1}{ }^{i}$ is the firing rate of cell ' 1 ' in trial ' $i$ ', $\bar{r}$ is the mean firing rate averaged across the trials, and $\sigma$ is standard deviation of the responses.

\section{Results}

\subsection{Adaptation increases gamma synchronization}

We used multi-contact laminar electrodes (Plextrode $\AA$ U-Probe, Plexon Inc.) to record neuronal activity at $20 \mathrm{~V} 1$ recording sites, each measured at 16 different depths, while two monkeys performed a rapid adaptation fixation task (Fig. 1a). While animals fixated a white dot at the center of a screen, an adapting stimulus was flashed for $300 \mathrm{~ms}$ in the center of the neurons' receptive field. After $100 \mathrm{~ms}$ of blank, a test stimulus of random orientation ( 8 equally spaced orientations spanning $0-180^{\circ}$ ) was presented for 300 ms.

The adapting stimulus was either a random dot patch (control condition) or a sine-wave grating with spatial characteristics identical to those of the test stimulus, but fixed in orientation (adaptation condition). In control trials, the presentation of the test stimulus led to pronounced synchronous activity across all cortical layers, with the most significant increase in gamma synchronization in the granular layers (Fig. 
2a). However, after adaptation, there was a significant increase in gamma synchronization specifically in the supragranular layers (Fig. 2b).

\subsection{Adaptation reduces neuronal correlations}

Responses to dynamic test stimuli in V1 of fixating monkey were recorded before and after brief (400-ms) adaptation to a sinewave grating of fixed orientation (Fig 3a). We used a movie sequence as test stimulus, in which each frame was a sine-wave grating of pseudorandom orientation flashed at $60 \mathrm{~Hz}$. We measured noise correlations between pairs of nearby neurons ( $n=423$ pairs).

Fig. $3 \mathrm{~b}$ shows an example of a pair of cells preferring nearby orientations that exhibit a strong reduction in correlations after adaptation (the pre-adaptation condition is labeled 'control'). Across the population, we found an overall post-adaptation decrease in the absolute correlation coefficients. Subsequently, we computed network accuracy by estimating Fisher Information (FI) as the upper limit with which any decoding mechanism can extract information about stimulus orientation $[18,19]$. FI was computed by assuming that adaptation changes (i) only mean correlations, and (ii) both the mean and variability of correlations. Fig. 4a shows that whereas the post-adaptation reduction in mean correlations caused a $25 \%$ improvement in the network orientation discriminability threshold, taking into accounts both the changes in the mean and variability of correlations improved the post-adaptation discrimination threshold by $40 \%$.

As shown in Fig. 4b, brief adaptation caused an almost four-fold increase in FI when the network discriminated stimuli of similar and largely dissimilar orientation relative to the adapting stimulus, and a three-fold increase in FI for the discrimination of intermediate orientations.
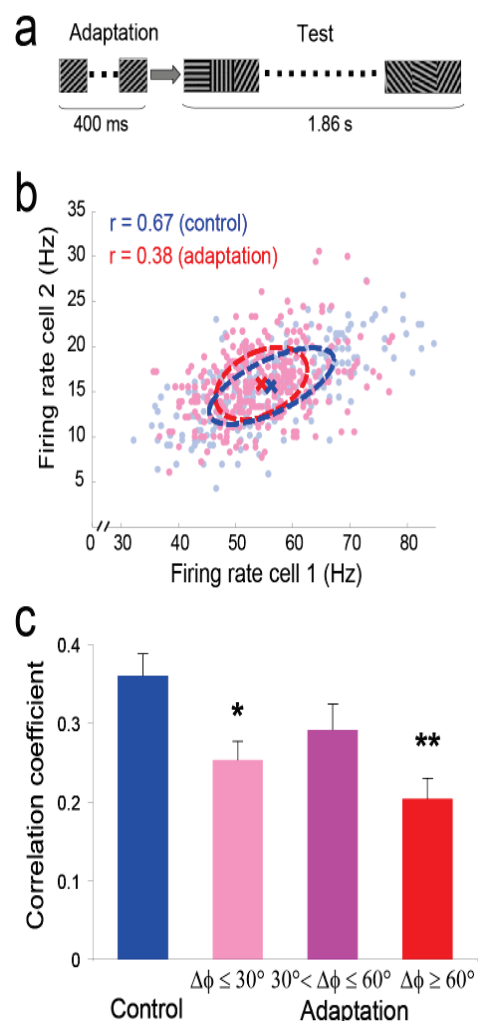

Fig. 3. Adaptation-induced response decorrelation in V1. (a) Schematic representation of the stimulus sequence: an adapting stimulus of fixed orientation was presented for $400 \mathrm{~ms}$ and was followed by a 60 -

$\mathrm{Hz}$ test stimulus of random orientation presented for $1.86 \mathrm{~s}$. (b) Scatter plot showing the trial-by-trial responses of two cells recorded simultaneously. Each dot represents the firing rates of both cells in a given trial. The dotted ellipses represent the two-dimensional gaussian fits of the firing rate distributions during control and adaptation. (c) The reduction in the mean correlation coefficients after adaptation depends on the adapting orientation. All panels are based on the correlation analysis of $\mathrm{n}=423$ cell pairs.

Error bars represent s.e.m. $(* \mathrm{P}<0.005$;

$$
* * \mathrm{P}<0.0005) .
$$




\section{Discussions and future directions}

Theoretically, adaptation has been proposed to reduce redundancy in sensory neurons, possibly by decorrelating responses, to improve coding efficiency $[20,21]$. However, besides the lack of experimental support, theories proposing the decorrelation hypothesis were unable to predict the changes in correlations across the entire network engaged in sensory computations

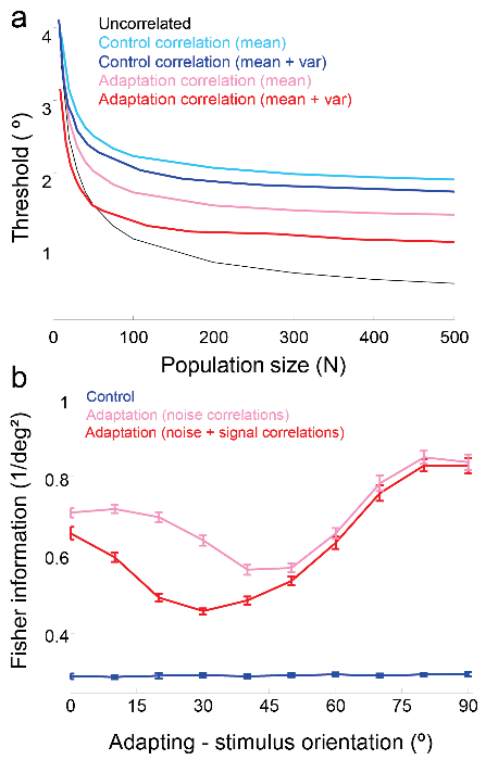

Fig. 4. Rapid adaptation enhances the accuracy of population coding. (a). Light blue/red curves: adaptation changes only the mean correlation coefficients; Dark blue/red curves: adaptation changes both the mean and variability of correlations. (b) The accuracy of population coding (FI) depends on $\Delta \theta$ and the adapting orientation (fixed at $0^{\circ}$; abscissa represents the test orientation). In control trials, FI is independent on the test orientation. Combining the effects of noise and signal correlations yields a more pronounced U-shape profile of FI.

We provide empirical evidence that adaptation causes both a selective reduction in the strength and variability of correlations and an improvement in population coding efficiency. These results are consistent with the 'efficient coding hypothesis' [22,23], i.e., sensory neurons are adapted to the statistical properties of the stimuli they are exposed to, and with psychophysical changes in human discrimination performance after adaptation. We argue that the visual system employs a metabolically inexpensive solution (selective decorrelation) to adapt neural responses to the statistics of the input stimuli and improve coding efficiency.

For instance, theoretical studies have suggested that temporally decorrelated inputs are transmitted less efficiently than correlated inputs [22]. In addition, our finding that neurons in the supragranular layers exhibit the largest increase in gamma synchronization after adaptation and the highest correlation with the post-adaptation improvement in feature coding has functional implications for models of cortical function. Indeed, neurons in the supragranular layers of $\mathrm{V} 1$ provide the only cortical input to downstream visual areas. Intragranular layers also constitute output layers, but they target deep subcortical structures such as the thalamus and superior colliculus. Therefore, neurons in higher-order cortices would benefit most if cells in the supragranular layers would exhibit a large increase in stimulus coding after adaptation.

\section{Acknowledgments}

Supported by the NIH EUREKA Program, National Eye Institute, the Pew Scholars Program, the James S. McDonnell Foundation (V.D.).

\section{References}

[1] Dragoi V, Sharma J, Miller EK, Sur M (2002) Dynamics of neuronal sensitivity in visual cortex and local feature discrimination. Nat Neurosci 5 (9):883891.

[2] Felsen G, Shen YS, Yao H, Spor G, Li C, Dan Y (2002) Dynamic modification of cortical orientation tuning mediated by 
recurrent connections. Neuron 36 (5):945954.

[3] Dragoi V, Sur M (2006) Image structure at the center of gaze during free viewing. J Cogn Neurosci 18(5):737-748.

[4] Muller JR, Metha AB, Krauskopf J, Lennie P (1999) Rapid adaptation in visual cortex to the structure of images. Science 285 (5432):1405-1408.

[5] Hansen BJ, Eagleman S, Dragoi V (2011) Examining Local Network Processing using Multi-contact Laminar Electrode Recording. J Vis Exp (55).

[6] Nassi JJ, Callaway EM (2009) Parallel processing strategies of the primate visual system. Nat Rev Neurosci 10 (5):360-372.

[7] Hansen BJ, Dragoi V (2011) Adaptation-induced synchronization in laminar cortical circuits. Proc Natl Acad Sci U S A.

[8] Lakatos P, O'Connell MN, Barczak A, Mills A, Javitt DC, Schroeder CE (2009) The leading sense: supramodal control of neurophysiological context by attention. Neuron 64 (3):419-430.

[9] Engel AK, Konig P, Kreiter AK, Singer W (1991) Interhemispheric synchronization of oscillatory neuronal responses in cat visual cortex. Science 252 (5010):11771179.

[10] Fries P, Reynolds JH, Rorie AE, Desimone R (2001) Modulation of oscillatory neuronal synchronization by selective visual attention. Science 291 (5508):1560-1563.

[11] Gray CM, Konig P, Engel AK, Singer W (1989) Oscillatory responses in cat visual cortex exhibit inter-columnar synchronization which reflects global stimulus properties. Nature 338 (6213):334-337.

[12] Freiwald WA, Kreiter AK, Singer W (1995) Stimulus dependent intercolumnar synchronization of single unit responses in cat area 17. Neuroreport 6 (17):2348-2352. [13] Cardin JA, Carlen M, Meletis K, Knoblich U, Zhang F, Deisseroth K, Tsai LH, Moore CI (2009) Driving fast-spiking cells induces gamma rhythm and controls sensory responses. Nature 459 (7247):663667.

[14] Chalk M, Herrero JL, Gieselmann MA, Delicato LS, Gotthardt S, Thiele A (2010) Attention reduces stimulus-driven gamma frequency oscillations and spike field coherence in V1. Neuron 66 (1):114125. 